Matthias Pilz/Thomas Deißinger

\title{
SYSTEMVARIANTEN BERUFLICHER QUALIFIZIERUNG: EINE SCHOTTISCH-ENGLISCHE VERGLEICHSSKIZZE IM ZEICHEN DER MODULARISIERUNGSDEBATTE
}

\begin{abstract}
Summary: The problem which generates the motivation for comparing VET systems can be seen in the present and refers to the fact that the vocational education and training systems of Europe have one common trait: They differ sharply from one another. The differences in education and training between nations are not always apparent at first sight but surely well up whenever it comes to European integration. The question is whether this may also be said when looking at "intra-national“ comparisons or „home internationals“. In the case of England and Scotland ,individual" training infrastructures may also be traced back to and reconstructed from deep-rooted traditions. On the other hand, the two countries' „educational landscapes“ in general appear similar at first sight while two common features of the VET systems strike the eye: the firm belief that companies should be responsible for training and, secondly, the well-documented inclination to implement and even enforce a policy of establishing national qualification frameworks based on modular principles and linked to competence-based assessment. Against this background the article depicts some of the major differences between the two „countries“.
\end{abstract}

\section{EINFÜHRUNG}

Das Bildungssystem in Großbritannien stellt sich für den ausländischen Beobachter auf den ersten Blick als ein uniformes System dar. Strukturen, Bildungsprogramme und Begrifflichkeiten erscheinen gleich oder zumindest sehr ähnlich. Dies führt dazu, dass bei international-vergleichenden Untersuchungen nicht selten von den englischen Gegebenheiten auf Großbritannien bzw. das gesamte Vereinigte Königreich geschlossen wird und so der Eindruck entsteht, in England und Schottland seien homogene Bildungsstrukturen anzutreffen (Brand, 1997). Der Verzicht auf eine präzise Differenzierung von Strukturen und Konzepten beider Landesteile, wie er sich nicht zuletzt in einfachen Fußnoten-Verweisen manifestiert (Koch/Reuling, 1997, S. 4), kann jedoch mitnichten befriedigen, denn es „... sind die Unterschiede so groß, dass eine ernsthafte Untersuchung des Bildungsund des Ausbildungswesens im Vereinigten Königreich, die sich allein auf die 
Situation in England und Wales beschränkt (was häufig der Fall ist), kein wahrheitsgetreues Bild vermitteln würde“ (Twining, 1994, S. 10). Selbst im Rahmen britischer Forschungsprojekte wird Schottland (und teilweise auch Wales) vielfach nur eine geringe oder gar keine Beachtung geschenkt (Raffe, 1991, S. 47; Raffe et al., 1999a, S. 69; Raffe et al., 1999 b, S. 9 f. u. S. 18)' ${ }^{1}$ Erst in letzter Zeit lenken immer mehr britische Bildungsforscher ihr Interesse auf den intranationalen Vergleich, d.h. auf den Aspekt der sog. „Home Internationals in Comparative Research" (Raffe, 1991; Raffe et al., 1999 a; Raffe et al., 1999 b, S. 1822).

Gründe für diese relativ einseitige Fokussierung der bildungsstrukturellen und bildungspolitischen Gegebenheiten auf den britischen Inseln sind sicherlich in der langjährigen politischen Dominanz Londons in Bildungsangelegenheiten zu sehen (Raffe, 1998, S. 595 f.), die sich erst seit der Einräumung von Autonomierechten für die einzelnen Landesteile zu relativieren scheint (Scottish Parliament, 2001). Des weiteren sorgt das im Verhältnis zu England geringere Gewicht Schottlands bei der Bevölkerungszahl dafür, dass in der Öffentlichkeit die gesamtstaatliche Bedeutung regionaler Innovationen im Bildungssektor als eher gering angesehen wird $^{2}$. Schließlich hat vermutlich die komplexe Struktur des britischen Bildungssystems, dessen berufliches Segment in der Vergangenheit immer wieder - nicht zu Unrecht - als „Qualifikationendschungel“ bezeichnet wurde (Greuling, 1996, S. 77 f.), ihren Anteil an dieser Perzeption. Was objektiv als eine notorisch hohe Innovationsfrequenz bei der Initiierung berufsbildungspolitischer Reformprojekte erscheinen mag, wirft für den forschenden Beobachter nicht unerhebliche Komplexitätsprobleme auf, die sich nicht nur auf das partielle „Anpassungschaos“ (Pilz, 1999 a, S. 290 f.; Pilz, 2000, S. 15) erstrecken, sondern auch die Nomenklatur der staatlichen und halbstaatlichen Programme bzw. Initiativen (Gunning, 1999) einschließen. Somit verwundert nicht, wenn bei ausländischen Experten eine gewisse Antipathie gegenüber etwaigen „Komplexitätserweiterungen“ durch Hinzunahme beispielsweise des „Sonderfalls Schottland“ in berufsbildungspolitische und berufspädagogische Untersuchungen entsteht.

In diesem Aufsatz soll der Versuch unternommen werden, einige markante Unterschiede der strukturellen Facetten des beruflichen und - wo für das Verständnis notwendig - auch des allgemeinen Bildungssystems Englands und Schottlands i.S. einer einfachen ,Juxtaposition“, d.h. eines Nebeneinanderrückens homologer Merkmalsdimensionen (Hilker, 1962; S. 106 f.; Schriewer, 1987, S. 631-633), herauszuarbeiten ${ }^{3}$. Für den Praktiker mag die Zweckmäßigkeit eines solchen Vorgehens darin liegen, diese identifizierten Alteritäten als unmittelbare Orientierungshilfe zu nutzen, wenn es beispielsweise um die Vorbereitung von Schüleraustauschprogrammen und Schulpartnerschaften in einem der beiden Lan- 
desteile geht ${ }^{4}$. Aus wissenschaftlicher Sicht stellen sich jedoch zunächst folgende prinzipielle Fragen:

- Das britische Berufsbildungssystem wird im Allgemeinen als ein von betrieblichen Interessen und betrieblichen Handlungslogiken bestimmtes Gebilde charakterisiert. Man könnte, wie Greinert dies getan hat, von einem „Marktmodell beruflicher Qualifizierung“" sprechen (Greinert, 1988), oder (was wir für zutreffender halten) von einem ,funktionsorientierten Qualifizierungsstil“ ausgehen, weil mit dieser Kennzeichnung die offensichtliche Orientierung von Qualifizierungsinhalten an Arbeitsplätzen, nicht an „Berufen“, zum Ausdruck gebracht werden kann (näheres hierzu bei Deißinger, 1995; 1999). Die Frage, die sich hierbei ad hoc stellt, ist die nach der Relativierung dieser betrieblichen Autonomie durch institutionelle Innovationen sowie bürokratische (nicht notwendigerweise staatliche) Überformungen des Systems, wie sie seit den achtziger Jahren zu beobachten sind. Möglicherweise lassen sich in diesem $\mathrm{Zu}$ sammenhang regionale Differenzierungen und Akzentuierungen in den beiden Landesteilen identifizieren, die zweifelsohne auf differente historisch-kulturelle Prägungen verweisen würden.

- Gegen eine solche Vermutung spricht jedoch, dass sich das Vereinigte Königreich insgesamt in den letzten fünfzehn Jahren gleichsam als „Musterland“ für eine flächendeckende und ausgesprochen radikal realisierte Politik der Modularisierung beruflicher Bildung im Kontext des sog. „lebenslangen Lernens“ profiliert hat (Deißinger, 2001 a). Diesem Umstand wird u.a. im Weißbuch der Europäischen Kommission aus dem Jahre 1996 Rechnung getragen (Kommission der EG, 1996), nachdem bereits 1992 der Rat der EU in einer Richtlinie die Bedeutung des britischen Zertifizierungssystems für Formalisierungsbemühungen im Zusammenhang mit der wechselseitigen Anerkennung nationaler Berufszertifikate herausgestellt hatte (Rat der EG, 1992, S. 26). Deutsche Berufspädagogen nehmen dies zum Anlass, dem Modulsystem als einem ,auf europäischer Ebene vorteilhaften Modell der Berufsbildung mit hohem Flexibilitätspotential“ eine besondere „Zukunftsfähigkeit“ zu attestieren (Münk, 1995, S. 35; Geißler, 1991). Hierbei wird jedoch - dies macht die Notwendigkeit einer systematisch-komparativen Betrachtung deutlich - pauschal von einer einheitlichen Konzeption innerhalb des Vereinigten Königreichs ausgegangen - eine Fehleinschätzung, die die Analyse der schottischen berufspädagogischen Verhältnisse zurecht rücken möchte.

- Letztlich dürfte insbesondere aus deutscher Perspektive die Interdependenz zwischen gesamtstaatlichen Interessen und Zwängen einerseits sowie andererseits den Ansprüchen und Ausformungen von föderal organisierten Bildungs- 
strukturen von Interesse sein. Am Beispiel der erst kürzlich erweiterten Autonomieregelungen in Schottland lassen sich möglicherweise Einflussgrößen und Tendenzen aufzeigen, die der Entstehung eines regional begrenzten und völlig eigenständigen (Berufs-)Bildungssystems entgegenstehen.

\section{ZUR UNTERSCHIEDLICHKEIT DES SCHOTTISCHEN UND ENGLISCHEN SCHULSYSTEMS}

Das schottische Bildungssystem hatte sich bereits vor der Vereinigung mit England im Jahre 1707 sowohl auf rechtlicher Ebene als auch in der Praxis als ein Gebilde mit eigenem Gesicht etabliert (Scotland, 1969; Clark, 1997 a, S. 3 f.). Diese Eigenständigkeit konnte bis heute in vielen Bereichen aufrecht erhalten werden und hat zu von England abweichenden Ausprägungsformen im Bereich der staatlich verantworteten schulisch-vorberuflichen Bildung geführt. Divergenzen zeigen sich beispielsweise in der unterschiedlichen Gestaltung der Lehreraus- und -weiterbildung: Mit dieser Aufgabe ist in Schottland der 1966 gegründete verbandsähnliche General Teaching Council for Scotland (1995) betraut (Clark, 1997 b; Humes/ Bryce, 1999), während in England diese Funktion vornehmlich direkt von staatlichen Institutionen übernommen wird. Allerdings ist mit der 1991 initiierten Gründung eines General Teaching Council for England und der 1998 vorgenommenen rechtlichen Verankerung eine Nivellierung der beiden Systeme absehbar (Whitburn, 1995; Nitschke, 1986, S. 98 f.). Ob allerdings damit auch eine Aufwertung des Lehrerberufs in England auf das in der schottischen Gesellschaft verankerte Niveau erfolgen wird, bleibt abzuwarten (Raffe et al., 1999 b, S. 17).

Primarstufe und Sekundarstufe I

In Schottland erfolgt die Einschulung wie in England mit fünf Jahren. Die schottische Sekundarstufe I ist nach Durchlaufen der sieben Klassen der Primary School vierstufig als Secondary School organisiert. Oftmals sind jedoch Primar- und Sekundarschule in einem Schulzentrum zusammengefasst. Am Ende der Sekundarstufe I erwerben die Schüler das Scottish Standard Grade Certificate (Scottish Office, 1996; Mitchell, 1995, S. 38-40; Boyd, 1997, S. 59-62). Die besondere Bedeutung eines für alle Schüler qualitativ hochwertigen schottischen Sekundarschulbereichs hat u.a. Boyd (1997, S. 53) herausgestellt, der konstatiert: ,... the efforts of secondary schools to have a positive ethos, to achieve high standards of attainment for all and to become more, effective' make them the envy of the rest of the United Kingdom.“ 
Die Curricula und der Umfang des Fächerangebots in den Sekundarschulen werden weitgehend eigenständig von den lokalen Bildungsbehörden und den Schulleitungen bestimmt, wobei der zentral operierende Scottish Consultative Council on the Curriculum diese Subsidiarität nur durch einige Richtlinien einschränkt (Eurydice Unit Scotland, 1995, S. 454; Clark, 1997 a, S. 9). Im Gegensatz zu den englischen Eltern haben die schottischen über die school boards sowohl auf das Curriculum als auch auf die finanzielle Mittelverteilung in den Schulen erheblichen Einfluss (Munn, 1997, S. 129 f.). Inhaltlich können für das schottische Curriculum jedoch kaum kulturspezifische Besonderheiten i.S. einer „scottishness of the system“ (Boyd, 1997, S. 64) ausgemacht werden. Lediglich die fakultative Vermittlung der gälischen Sprache stellt in diesem Bereich eine Besonderheit dar (Harrison, 1997).

In England ist die Primary School ab dem Alter von fünf Jahren im Umfang von sechs Klassen (2 Jahre Infant School und 4 Jahre Junior School) zu besuchen. Dafür besteht die anschließende obligatorische Secondary School aus fünf Klassenstufen. Am Ende der Sekundarstufe I erhalten die Schüler das General Certificate of Secondary Education (GCSE), welches sich von den schottischen Standard Grades durch eine stärkere rechtliche Reglementierung und damit Uniformisierung über das in ganz England verbindliche National Curriculum unterscheidet (Artzfeld, 1997, S. 2946 f.; Twining, 1994, S. 55). Während in Schottland fast alle Schüler eine Gesamtschule besuchen, bestehen neben diesen in England auch Schulformen mit selektiver Komponente (grammar/secondary modern/technical schools) (Mackinnon/Stratham/Hales, 1996, S. 76 f.). Außerdem hat sich hier als Ergebnis der „marktorientierten“ Bildungspolitik der achtziger Jahre (Saran, 1988) mittlerweile eine bedeutend größere Zahl staatlicher Schulen als in Schottland von der direkten staatlichen Lenkung losgesagt. Diese Schulen erhalten nur noch ein Globalbudget, mit dem sie eigenständig wie ein Unternehmen arbeiten können (Clark, 1997 a, S. 5 f.; Munn, 1997, S. 131; Raffe et al., 1999 b, S. 13 f. sowie S. 17).

Die Überwachung aller Schulen obliegt in England dem Office of Standards in Education und in Schottland dem mit hiervon etwas abweichenden Aufgaben und Kompetenzen betrauten Her Majesty's Inspectorate (Raffe et al., 1999 b, S. 12; Mitchell, 1995, S. 41-43; Clark, 1997 a, S. 14 f.). Privatschulen spielen in Schottland mit einem Schüleranteil von unter fünf Prozent eines Altersjahrgangs im Gegensatz zu England nur eine geringe Rolle (Eurydice Unit Scotland, 1995, S. 448) ${ }^{5}$. Während diese in England als Public Schools bezeichnet werden, tragen sie in Schottland den Namen Independent Schools. Public Schools sind deshalb hier ausschließlich Schulen in staatlicher Trägerschaft (Mitchell, 1995, S. 11). 


\section{Sekundarstufe II}

Die Sekundarstufe II besteht in Schottland aus einer oder zwei Klassenstufen. Abgeschlossen wird die Oberstufe i.d.R. mit der Scottish Certificate Examination at Higher Grade, die den Zugang zu den Hochschulen ermöglicht. Diese sog. Highers werden in drei bis fünf Fächern abgelegt und basieren auf einem Curriculumanteil von jeweils 160 Unterrichtsstunden. Leistungsstarke Schüler verlassen die Oberstufe vielfach bereits nach dem ersten Oberstufenjahr. Die Benotung der Highers erfolgt von A für sehr gute Leistungen bis D, was das Nichtbestehen dokumentiert. Die nur einjährige Oberstufe wird von diversen Kritikern als zu kurz hinsichtlich des Spektrums und der Tiefe der zu vermittelnden Fähigkeiten und Kenntnisse sowie als pädagogisch problematisch mit Blick auf den vielfach zu beklagenden Schulstress (es wird dabei von einem two-term dash gesprochen, vgl. Raffe, 1993, S. 62) bezeichnet. Nach einem zweiten Schuljahr in der Oberstufe können die Schüler neben der Verbesserung der Noten und zusätzlichen Highers das Certificate of Six Years Studies erwerben, welches bei einigen Universitäten zur Anrechnung von Leistungen im Grundstudium berechtigt. An diesem Punkt erkennt man die für ein traditionsreiches Land wie Großbritannien doch auffällige Flexibilität bei der Abgrenzung von Bildungssystemsegmenten, die auch vor dem Hochschulbereich nicht Halt macht ${ }^{6}$. Im Übrigen hat in Schottland der Besuch der Oberstufe eine lange Tradition mit höheren Übergangsquoten von der Pflichtsekundarschule in den Sekundarbereich II als in England (Spours/Young, 1996, S. 11).

Von besonderem Interesse hinsichtlich einer friktionsfreien Mobilität von Studienanfängern ist der Umstand, dass englische Universitäten die schottischen Highers als Zugangsberechtigung anerkennen, wie umgekehrt die schottischen Hochschulen die englischen A-Levels. Ein entscheidender Unterschied zwischen den Hochschulen stellt das schottische Honours Degree dar. Dieser hochwertige und spezialisierte Abschluss kann nach vier Studienjahren an schottischen Universitäten erworben werden und baut auf dem nach drei Jahren abzulegenden Degree auf (Scottish Office, 1995). Da in England die universitäre Ausbildung hingegen i.d.R. nur drei Jahre dauert und mit dem Degree abschließt, genießen die renommierten schottischen Universitäten bei englischen Studenten eine hohe Attraktivität.

Mit Beginn des Schuljahres 1999/2000 wurde in Schottland erstmals ein auf modularer Basis konzipiertes allgemeine und berufliche Bildungsgänge umfassendes einheitliches Konzept im schulischen Sekundarbereich realisiert, welches den Programmnamen Higher Still trägt (ausführlich hierzu Pilz, 1999 c). Eine stärkere Verknüpfung von allgemeinem und beruflichem Lernen wird auch in England seit den neunziger Jahren forciert, allerdings sind die dortigen Strukturen der- 
zeit im Gegensatz zu Schottland noch durch weitgehend separierte allgemeine und berufliche Bildungsgänge gekennzeichnet (Spours et al., 1998; Raffe et al., 1998 a; Raffe et al., 1999 a; Raffe, 1997, S. 73-79; Howieson et al., 1997).

In England besteht die Oberstufe an den allgemeinbildenden Schulen verpflichtend aus zwei Klassenstufen, die mit dem General Certificate of Education at Advanced Level (GCE-A) abgeschlossen werden. Sie wird an einer siebenstufigen Gesamtschule (mehr als die Hälfte aller Gesamtschulen verfügt über eine Oberstufe) oder an separierten zweistufigen Sixth Form Colleges absolviert, die allerdings heute formell dem Bereich der Further Education zugerechnet werden (Mackinnon/Stratham/Hales, 1996, S. 78). Die A-Levels sind vom Stundenumfang her betrachtet gewichtiger und inhaltlich weniger fachspezifisch ausgelegt als die schottischen Highers, was sich auch darin zeigt, dass in England i.d.R. maximal nur drei A-Levels erworben werden. Die Notenskala setzt sich bei den A-Levels aus sieben Noten (A für ,sehr gut“ bis U für ,keine Note“, was dem deutschen ,ungenügend" entspricht) zusammen.

In England besteht die Besonderheit, dass neben den allgemeinbildenden Schulen auch Colleges of Further/Higher Education mit regional differierender Ausgestaltung und Schwerpunktsetzung allgemeinbildende schulische wie auch berufliche Abschlüsse - durchaus auch i.S. eines „zweiten Bildungsweges“ für Jugendliche und Erwachsene - offerieren. Tatsache ist, dass über die Hälfte der englischen Jugendlichen nach dem 16. Lebensjahr (dem Ende der Schulpflichtzeit) nicht an Schulen, sondern an Colleges weitere Abschlüsse anstrebt. In Schottland sind die Colleges hingegen weitaus stärker auf die Weiterbildung und die Berufsbildung ausgerichtet, was sich in geringeren Übergangsquoten von den Sekundarschulen als im englischen Landesteil ausdrückt (Raffe et al., 1999 b, S. 14). Als eine weitere Besonderheit des englischen Subsystems sind die sog. General National Vocational Qualifications (GNVQs) anzusehen, die eine berufsorientierte Option in den Sekundarschulen verkörpern, mit der die Regierung die Gleichwertigkeit von allgemeiner und beruflicher Bildung - bis hin zum Hochschulzugang unterstreichen möchte (Hodgson/Spours, 1997). Zwar bestand mit den General Scottish Vocational Qualifications (GSVQs) in Schottland ein vergleichbares Bildungsangebot, das allerdings keine größere Bedeutung gewinnen konnte (Pilz, 1999 a, S.130-133; Pilz, 1999 c, S. 333).

\section{Berufliche Bildung im INTRANATIONALEN Vergleich}

Besonderes Interesse verdient Großbritannien für den deutschen Beobachter durch den Sachverhalt, dass die dortige Berufsbildungspolitik gegenwärtig versucht, tra- 
ditionelle Formen wie die Lehrlingsausbildung sowie „moderne“ Konzepte, die dem Modell des competence-based learning folgen, miteinander $\mathrm{zu}$ verbinden. Tatsache ist jedoch, dass die Lehre in den neunziger Jahren wie auch in der Gegenwart nur noch einen Rest- und Randbereich des britischen Ausbildungssystems verkörpert (Marsden/Ryan, 1991, S. 259 ff.). Ryan beziffert für 1998 die Teilnehmer an einer apprenticeship (hierbei muss es sich nicht notwendigerweise um eine traditionelle Lehre handeln), in Großbritannien insgesamt auf rund 190.000, wobei in England and Wales die Zahl derjenigen, die eine sog. ,moderne Lehrlingsausbildung“ (Modern Apprenticeship) durchlaufen, allein ca. 120.000 beträgt. Verglichen mit dem deutschen dualen System deuten diese Zahlen dennoch auf ein eher marginalisiertes Subsystem der Berufsausbildung hin (Ryan, 2001).

Bei der Modern Apprenticeship (Unwin/Wellington, 1995; Fuller/Unwin, 1998) handelt es sich um eine der interessantesten Innovationen der britischen Berufsbildungspolitik. Jedoch verkörpert sie nicht einfach nur eine Renaissance der traditional apprenticeship (Snell, 1996, S. 319), da sich zwei Grundzüge der berufsbildungspolitischen Orientierung der Thatcher-Zeit (Deißinger/Greuling, 1994) in ihr manifestieren: Zum einen ist sie eine „staatlich geförderte Initiative zur Belebung des Ausbildungssystems" (Ertl, 1998, S. 171); zum zweiten ist sie an das System der nationalen Befähigungsnachweise (National Vocational Qualifications u. Scottish Vocational Qualifications, siehe nachfolgende Ausführungen) angekoppelt, wobei die Absolventen mindestens eine NVQ/SVQ auf Level 3 erreichen sollen. Ein weiterer Unterschied zur Lehre alter Prägung und damit auch zu jener im dualen System in Deutschland besteht darin, dass zwar Lehrverträge abgeschlossen werden, jedoch keine verbindlichen Ausbildungszeiten festgelegt werden müssen.

Nicht vergessen werden darf hierbei jedoch, dass es sich hier lediglich um ein Subsystem des heterogenen britischen Ausbildungswesens handelt. Im wesentlichen bestimmen Institutionen, die von der Wirtschaft dominiert werden, nach wie vor die Kontextbedingungen, unter denen in Großbritannien berufliche Bildung stattfindet. Daran vermochten auch die Reformmaßnahmen der achtziger Jahre nichts zu ändern, die vorrangig auf das Finanzierungsproblem und kurzfristige Bemühungen, die Allokationsfunktion beruflicher Qualifizierung zu verbessern, ausgerichtet waren. Auf die betrieblichen Ausbildungssegmente entfallen vor diesem Hintergrund rund 47\% der Erstausbildungsteilnehmer. In der Vollzeitvariante besuchen immerhin 35,7\% und in der Teilzeitvariante 13,6\% aller Erstausbildungsteilnehmer im Vereinigten Königreich ein College of Further Education. Hinzu kommt mit 4,6\% die schulische Berufsbildung, die in den Schulen des allgemeinbildenden Schulsystems in Form von GNVQs/GSVQs (allgemeinen nationalen beruflichen Qualifikationen) vermittelt wird (Kommission der EG/EUROSTAT/CEDEFOP, 1997, S. 62). 
Folgende Merkmale lassen sich zusammenfassend für das britische Ausbildungssystem anführen:

- die weitgehende Freiheit der Betriebe bei der Gestaltung von (innerbetrieblichen) Berufsausbildungsmaßnahmen;

- die Vielfalt unterschiedlichster Träger und Lernorte der Berufsausbildung;

- die Offenheit der Berufsausbildung gegenüber Sekundarbildung und Weiterbildung sowie

- der fakultative Charakter standardisierter Befähigungsnachweise.

Kern des britischen Berufsbildungsverständnisses ist hierbei die Verbindung von competence-based learning und Modularisierung (Wolf, 1995; Mansfield, 1990; Williams/Raggatt, 1998). In ihm verbindet sich auch augenfällig die Berufsbildungspraxis in den beiden großen Landesteilen. So werden seit 1984 in Schottland berufliche Qualifikationen in modularer Form an Schulen, Colleges (beruflichen Schulen), sonstigen Bildungseinrichtungen und in Unternehmen offeriert. Diese als National Certificate Modules (NC-Modules) bezeichneten Qualifizierungsmaßnahmen haben eine standardisierte Länge von jeweils 40 Unterrichtsstunden und werden diskret geprüft und zertifiziert. Lernziele, Lerninhalte und Prüfverfahren sind in einem „Modulsteckbrief“ dezidiert festgelegt (Pilz, 1999 a, S. 116119 sowie S. 145 f.). Ein derart radikales Verständnis von Modularisierung ist in England nicht realisiert worden (Pilz, 1999 a, S. 145 f. sowie S. 155 f.). Mit Einführung des Higher Still-Programms wurden die NC-Module jüngst Teil eines neuen Gesamtsystems allgemeiner und beruflicher Bildungsoptionen auf der Sekundarstufe. Raffe verweist darauf, dass es sich durchaus um einen „broad vocational track" in Schottland und England handle (Raffe, 2000, S. 14): Er werde durch die englischen GNVQs einerseits und die schottischen Modulqualifikationen, die neben den Highers angeboten werden, repräsentiert. Allerdings sei das englische System institutionell deutlich heterogener und flexibler als das schottische, auch wenn sich beide wiederum durch die Grenzziehung zwischen Schulen und Colleges auszeichneten (Raffe, 2000, S. 14 f.).

Derzeit nicht direkt von Higher Still betroffen ist das System der 1989 eingeführten Scottish Vocational Qualifications (SVQs). Diese aus i.d.R. sechs bis zehn verschiedenen Modulen (entsprechend den NC-Modulen) bestehenden Ausbildungsangebote werden in elf verschiedenen Tätigkeits- und Qualifizierungsbereichen, z.B. in der Land- und Forstwirtschaft oder im kaufmännischen Sektor, zugänglich gemacht, wobei es sich um „Gesamtqualifikationen“ auf fünf Niveaus handelt. SVQs werden vornehmlich von den Unternehmen genutzt, nicht nur, weil sie maßgeblich an der Entwicklung dieser Ausbildungsgänge beteiligt sind, son- 
dern weil erstere betriebliche Einsatzbedingungen - vor allem in technisch-infrastruktureller Hinsicht - voraussetzen.

Das englische Pendant zu den SVQs sind die National Vocational Qualifications (NVQs), die sich trotz der anderen Namensgebung nur in einigen institutionellen Aufgabenzuweisungen von den SVQs unterscheiden ${ }^{7}$. Der Grund für den hohen Grad an Uniformität, der z.B. durch die fünfgliedrige Niveaustufenskala sowie die Feingliederung der Programme hinsichtlich Lernzielen und Leistungskriterien dokumentiert wird, ergibt sich aus dem Modellcharakter des englischen NVQ-Systems, das 1986 eingeführt wurde (Deißinger, 1994). Neben der Regierung in London haben auch Arbeitgeberverbände und national vertretene Unternehmen bei der Konzeption der SVQs in nivellierender Weise beim „Juniorpartner Schottland" Einfluss genommen. Dies geschah vor allem vor dem Hintergrund der Notwendigkeit von Mobilität und Transparenz auf einem einheitlichen Arbeitsmarkt im Vereinigten Königreich. Prinzipiell sind alle SVQs in England und alle NVQs in Schottland wechselseitig anerkannt. Eigenständige schottische SVQs, die speziell auf die dortigen Wirtschaftsverhältnisse abgestimmt sind, finden sich nur in wenigen Branchen, wie z.B. in der Whiskyindustrie (Pilz, 1999 b).

Jedoch ergeben sich Unterschiede im institutionellen Bereich, und zwar zunächst bei der Kompetenzzuweisung hinsichtlich der Zertifizierung, Registrierung und Überprüfung der SVQs bzw. NVQs. Während in Schottland fast ausschließlich die halbstaatliche Bildungsbehörde Scottish Qualifications Authority (SQA) diese Aufgaben übernimmt (vor der Higher Still-Initiative war dies der Scottish Vocational Education Council, Scotvec genannt), delegiert seit 1997 das englische institutionelle Pendant, die Qualifications and Curriculum Authority (QCA), diese Aufgaben an über hundert private Zeugniserteilungsstellen, die als Awarding Bodies bezeichnet werden (Humes/Bryce, 1999; Pilz, 1999 a, S. 125128). Dadurch ist in dieser Hinsicht das englische System weniger zentralistisch, aber auch unübersichtlicher organisiert (Deißinger, 1996, S. 197 f.; Deißinger, 1998, S. 230). Eine weitere strukturelle Alterität stellt das auch in der deutschen vergleichenden Wirtschafts- und Berufspädagogik stark diskutierte, Anfang der achtziger Jahre eingeführte Youth Training-Programm (YT) dar, welches Jugendlichen über öffentlich geförderte Trainingsmaßnahmen den Eintritt in das Beschäftigungssystem ermöglichen soll (Deißinger/Greuling, 1994, S. 136-140). Dieses ursprünglich sowohl in England als auch in Schottland eingeführte Programm wird in Schottland nun im Rahmen des Modulprogramms Skillseekers weitergeführt (Scotvec, 1995), wohingegen es in England im System der sogenannten National Traineeships aufgegangen ist (Gospel, 1998, S. 342).

Auf institutioneller Ebene gibt es im Bereich der beruflichen Bildung zudem eine weitere Besonderheit. Neben den national agierenden und von den Arbeitge- 
bern für die Belange der beruflichen Qualifizierung gegründeten National Training Organisations (NTOs, vormals Industry Training Organisations, vgl. Gunning, 1999) haben in Schottland die staatlich finanzierten Organisationen Scottish Enterprise (SE) und Highlands and Islands Enterprise (HIE) großen Einfluss auf die berufliche Bildung, da sie diverse Qualifizierungsprogramme finanziell fördern. Jedoch sind sie wie auch die englischen NTOs quasi übergreifende Organisationseinheiten, die keine Ausbildungskonzepte eigenständig kreieren, während letztere in Schottland in der Zuständigkeit der 22 Local Enterprise Companies (LECs) bzw. in England der von derzeit knapp 100 Training and Enterprise Councils (TECs) liegen. Im Gegensatz zu den TECs haben die schottischen LECs einen größeren Aufgabenbereich, der neben der beruflichen Bildung auch die regionale Wirtschaftsförderung umfasst (Scottish Enterprise, 1997; Employment Department Group, 1994, S. 15; Fairly/Lloyd, 1995; Fairly, 1990; 1995; 1996).

\section{SCHLUSSBETRACHTUNG}

Die hier vorgestellten Aspekte stellen nur einen Ausschnitt von Alteritäten in den Erscheinungsformen der Bildungs- und Berufsbildungssysteme der beiden großen britischen Landesteile dar. Dennoch zeigt sich an ihnen, dass der intranationale Vergleich nicht wenige differente Sachverhalte und Begrifflichkeiten aufzudecken vermag, die sich (wie das, was sie bezeichnen) überdies mit der für Großbritannien typischen hohen Geschwindigkeit verändern und weiter entwickeln und die somit bei einem deutschen Beobachter, der von vergleichsweise homogenen und konstanten Vorstellungen über die berufliche Bildung ausgeht, Befremden auslösen. Hinzu kommt der eine umfassende Analyse und Vergleichsarbeit erschwerende Tatbestand, dass die schottischen und englischen Bildungsprogramme einerseits verschiedenen Denominationen aufweisen, andererseits aber auch im Inhalt gleicher oder ähnlicher Begriffe Unterschiede verborgen liegen können. So dominierte bisher in Schottland der Begriff „Modul“, während in England schon seit längerer Zeit der Begriff „Unit“ gebräuchlich ist (SQA, 1999, S. 1; Gunning, 1999; Reuling, 1998, S. 271). Schlüsselqualifikationen tragen in England den Namen „Key Skills" und in Schottland „Core Skills“, obwohl sich die Inhalte nur darin unterscheiden, dass hierbei in Schottland neben den auch in England üblichen Qualifikationsbereichen „Rechnen“, „Kommunikation“ und „Informationstechnologie“ zusätzlich die Bereiche „Problemlöseverhalten“ und „Mitarbeiterumgang“ zugrunde gelegt werden (Raffe et al., 1998 b, S. 49 f.; Gunning, 1999).

Dennoch kann als übergreifende, wenn auch nicht durchgehend feststellbare Charakteristik für das schottische Berufsbildungssystem zum einen eine stärkere 
Konzentration der Aufgaben in den Händen weniger staatlicher Stellen ausgemacht werden, der in England eine heterogene institutionelle Vielfalt gegenüber steht (Raffe, 2000, S. 14) - insbesondere was die Awarding Bodies im Bereich beruflicher Bildung betrifft. Zum anderen spielt der Bildungsaspekt in Schottland eine größere Rolle als in England, wo der Aspekt der Qualifizierung im Vordergrund steht, was sich in höheren Verbleibsraten im schottischen Schulsystem nach Ende der Pflichtschulzeit und einer stärkeren Stellung breiter angelegter Qualifizierungsgänge mit höherer Gewichtung des Lernprozesses und geringerer Bedeutung der Outputorientierung manifestiert (Spours/Young, 1996). Dennoch provoziert auch hier - wie in England - die starke Fokussierung beruflichen Lernens auf partialisierte Lernwege in der wissenschaftlichen Diskussion ernst zu nehmende Kritik (Canning, 1998; Canning 2000; vgl. zu England auch Deißinger, 1996, 2001 b).

Trotz der aufgezeigten Unterschiede und der zunehmenden Bildungsautonomie in Schottland wird es auch in Zukunft einige Bestimmungsgrößen mit großer Relevanz für die Ausgestaltung eines funktional einheitlichen bzw. kompatiblen Bildungssystems in Großbritannien geben. So müssen zum einen der problemlose Schulwechsel und die Mobilität von Studienanfängern gewährleistet werden. Zum andern erfordert der einheitliche britische Arbeitsmarkt die Schaffung von strukturähnlichen Befähigungsnachweisen. Außerdem werden die in die Entwicklung von Ausbildungsprogrammen involvierten (zumeist englischen) Großunternehmen auch in Zukunft auf eine einheitliche Ausgestaltung drängen. Weiterhin darf nicht vergessen werden, dass sowohl in England als auch in Schottland einige wenige private Bildungsträger aus historischen Gründen eine starke Stellung besitzen und Qualifizierungsmaßnahmen in großem Stil offerieren. So prägen z.B. das City and Guilds of London Institute und der Business and Technology Educational Council das berufliche (Weiter-)Bildungssystem in England und Schottland in erheblichem Maße (Twining, 1994, S. 53). Nivellierend und damit "harmonisierend“" dürften sich überdies von der Zentralregierung in London für ganz Großbritannien entwickelte und finanzierte Programme an der Schnittstelle zwischen Arbeitsmarkt- und Bildungspolitik, wie z.B. das aktuelle New Deal-Programm (Mädler/Pilz, 1999; Behrens, 2000), auswirken. Dennoch stellt sich für die Zukunft die Frage, wie das schottische Bildungssystem im Bereich der Annäherung bzw. Verknüpfung von allgemeiner und beruflicher Bildung einen möglichst eigenständigen Charakter bewahren und dabei auch die gewonnene Bildungsautonomie stärker in die Praxis umsetzen kann.

Für die weitere Entwicklung und Interdependenz der beiden hier untersuchten Systeme werden deshalb zunehmend neue Erklärungsmuster zu generieren sein. Bisher konnte die abweichende Entwicklung des schottischen Bildungssystems relativ einfach durch die ,explanation by origin“ (Paterson, 1997, S. 138) i.S. einer 
geschichtlichen Prägung erklärt werden ${ }^{8}$. Damit verbunden sind Ansätze, die eine Interdependenz zwischen kultureller Identität und der Ausgestaltung des Bildungssystems festgestellt haben (Robertson-Wensauer, 1994). Daneben wurde die Notwendigkeit zur Gestaltung eigener Elemente im Bildungswesen als entscheidender Beweggrund identifiziert, um der automatischen, gleichsam „fremdbestimmten“ Übernahme englischer Konzepte und Reformen entgegen zu treten. Beispielhaft lässt sich die Implementierung der schottischen National Certificate Modules anführen, die Anfang der achtziger Jahre als eine ,präventive Gegenmaßnahme“" gegen die von der englischen Manpower Services Commission initiierte Technical and Vocational Education Initiative implementiert wurden (Paterson, 1997, S. 147; Deißinger/Greuling, 1994, S. 141 f.). Ein weiterer plausibler Erklärungsfaktor für die Unterschiede dürfte auch der Ehrgeiz der schottischen Bildungspolitiker sein, ihre Existenz und Wichtigkeit durch eigene Entwicklungen im Bildungssystem zu legitimieren und zu unterstreichen (Raffe, 1998, S. 599). Mit der jetzt vollzogenen partiellen politischen Separierung und der vollständigen Bildungsautonomie in Schottland sind diese Erklärungsmuster jedoch weitgehend obsolet geworden. Wie sich die beiden Systeme im Spannungsfeld von notwendiger Konvergenz und erwünschter Divergenz entwickeln werden, ist derzeit noch ungewiss.

\section{ANMERKUNGEN}

1 Ein britischer Bildungsbeamter bekannte, Erkenntnisse über die Unterschiede der einzelnen Bildungssysteme Großbritanniens ,are not something we get every day. (...). This is virgin territory" (zit. nach Raffe, 1998, S. 594).

2 Für Nordirland umschrieb ein Bildungsexperte dieses Problem mit den Worten: „You have to understand the scale. We are but a pimple on the face of the UK" (zit. nach Raffe, 1998, S. 596).

3 Schottland bietet sich im Gegensatz zu Nordirland und Wales für eine Untersuchung der Unterschiede zu England besonders an, da es (auch historisch) als am stärksten unabhängig von den Entwicklungen in England angesehen werden kann und damit hier bereits auf den ersten Blick die meisten Disparitäten vermutet werden können (vgl. Raffe et al., 1999 b, S. 17 u. Raffe, 1998, S. 596 f.).

4 Für ausführlichere Darstellungen in der deutschsprachigen Literatur sei auf die (nicht mehr taufrische) Beschreibung des englischen Berufsbildungssystems von Stipriaan/ Lauterbach (1995) sowie die vor dem Hintergrund deutscher Problemlagen vorgenommenen aktuellen und historischen Analysen zu England bzw. Schottland der beiden Autoren dieses Aufsatzes verwiesen (Pilz, 1999 a; 1999 b; 1999 c; 2000; Deißinger, 1992; 1994; 1996; 1998; 1999; 2001 a; 2001 b). 
5 Nicht ohne eine gewisse Süffisanz stellt Michael (1990, S. 49) mit Blick auf die englischen Privatschulen fest: „Die meisten Schotten glauben, dass die staatlichen Schulen die bestmögliche Erziehung vermitteln sollten und dass eine Privatschul-Bildung lediglich solchen Leuten dient, die auf diesem Wege für Kontakte bezahlen, die im späteren Leben von gesellschaftlichem Nutzen sein könnten.“

6 Ein jüngstes Beispiel ist die Einrichtung des University Vocational Awards Council, der sich mit der Vergleichbarkeit beruflicher und akademischer Abschlüsse im Rahmen modularisierter Bildungskonzepte befasst.

7 Ein britischer Bildungspolitiker verglich die SVQs und NVQs mit den baugleichen Automodellen Honda Accord und Rover 600 - ,under the skin it's basically the same car“ (zit. nach Raffe, 1998, S. 599).

8 Paterson (1997, S. 139) führt dazu aus: „So, according to this kind of answer to the question ,why independent?‘, Scotland has a separate system for governing education at the end of the twentieth century because it has always had one: autonomy today is explained by the simple fact of autonomy yesterday."

\section{LITERATUR}

Artzfeld, H. (1997). Das Bildungssystem im Vereinigten Königreich, in: ibv (Informationen für die Beratungs- und Vermittlungsdienste der Bundesanstalt für Arbeit), Nr. 45, S. 2943-2956.

Behrens, M. (2000). Understanding the Interplay: A comparison of personal agency and social structures in New Deal and JUMP [Paper zum Vortrag beim Internationalen Kongress zur Berufsbildungsforschung vom 21. bis 24.09.2000 an der Universität Göttingen], Guildford.

Boyd, B. (1997). The Statutory Years of Secondary Education - Change and progress, in: Clark, M.M./Munn, P. (Eds.), Education in Scotland - Policy and Practice from Preschool to Secondary, London, S. 52-66.

Brand, W. (1997). Modularisierung in Großbritannien - Grundzüge der National Vocational Qualifications (NVQ), in: Berufsbildung - Zeitschrift für Praxis und Theorie in Betrieb und Schule, 51. Jg., H. 43, S. 22-24.

Canning, $R$. (1998). The failure of competence-based qualifications: an analysis of workbased vocational education policy in Scotland, in: Journal of Education Policy, Vol. 13, No. 5, S. 625-639.

Canning, $R$. (2000). The Rhetoric and Reality of Professional Competence-based Vocational Education in Scotland, in: Research Papers in Education, Vol. 15, No. 1, S. 65-89.

Clark, M.M. (1997 a). Education in Scotland: Setting the Scene, in: Clark, M.M./Munn, P. (Eds.), Education in Scotland - Policy and Practice from Pre-school to Secondary, London, S. 1-18. 
Clark, M.M. (1997 b). The Teaching Profession - Its qualifications and status, in: Clark, M.M.IMunn, P. (Eds.), Education in Scotland - Policy and Practice from Pre-school to Secondary, London, S. 98-114.

Deißinger, Th. (1992). Die englische Berufserziehung im Zeitalter der Industriellen Revolution. Ein Beitrag zur Vergleichenden Erziehungswissenschaft, Würzburg.

Deißinger, Th. (1994). Das Reformkonzept der „Nationalen beruflichen Qualifikationen“Eine Annäherung der englischen Berufsbildungspolitik an das „Berufsprinzip“?, in: Bildung und Erziehung, 47. Jg., S. 305-328.

Deißinger, Th. (1995). Das Konzept der „Qualifizierungsstile“ als kategoriale Basis idealtypischer Ordnungsschemata zur Charakterisierung und Unterscheidung von „Berufsbildungssystemen“, in: Zeitschrift für Berufs- und Wirtschaftspädagogik, 91. Jg., S. 367-387.

Deißinger, Th. (1996). Modularisierung der Berufsausbildung. - Eine didaktisch-curriculare Alternative zum „Berufsprinzip“?, in: Beck, $K$. et al. (Hrsg.), Berufserziehung im Umbruch. - Didaktische Herausforderungen und Ansätze zu ihrer Bewältigung, Weinheim, S. 189-208.

Deißinger, Th. (1998). Beruflichkeit als „organisierendes Prinzip“ der deutschen Berufsausbildung, Markt Schwaben.

Deißinger, Th. (1999). Beruflichkeit als Zusammenhang. Ein Vergleich mit England, in: Harney, K./Tenorth, H.-E. (Hrsg.), Beruf und Berufsbildung. Situation, Reformperspektiven, Gestaltungsmöglichkeiten (40. Beiheft zur Zeitschrift für Pädagogik), Weinheim, S. 189-207.

Deißinger, Th. (2001 a). Different Approaches to Lifelong Learning in Britain and Germany: A Comparative View with regard to Qualifications and Certification Frameworks, in: Harney, K. et al. (Eds.), Lifelong Learning: One focus - different systems, Frankfurt a.M. [im Druck].

Deißinger, Th. (2001 b). Modularisierung und Beruflichkeit - Überlegungen zur Differenzierung eines vermeintlichen Gegensatzes, in: Reinisch, H./Bader, R./Straka, G. A. (Hrsg.), Modernisierung der Berufsbildung in Europa. Neue Befunde der berufs- und wirtschaftspädagogischen Forschung, Opladen, S. 195-204

Deißinger, Th./Greuling, O. (1994). Die englische Berufsbildungspolitik der achziger Jahre im Zeichen der Krise eines „Ausbildungssystems“, in: Zeitschrift für Berufs- und Wirtschaftspädagogik, 90. Jg., H. 2, S. 127-146.

Employment Department Group (1994). Berufsausbildung in Großbritannien - Übersicht, o.O.

Ertl, H. (1998). Modern Apprenticeship Scheme: Ein Neuanfang für die Lehre in England und Wales?, in: Kölner Zeitschrift für Wirtschaft und Pädagogik, 13. Jg., H. 25, S. 171-187.

Eurydice Unit Scotland (1995). Schottland, in: Europäisches Bildungsinformationsnetz Eurydice (Hrsg.), Strukturen der allgemeinen und beruflichen Bildung in der Europäischen Union, Brüssel, Luxemburg, S. 446-460.

Fairley, J. (1990). The Impact of the Scottish Enterprise Reforms on Vocational Education and Training, in: Regional Studies, Vol. 24, No. 4, S. 363-366. 
Fairley, J. (1995). Training Strategy in Scotland: Co-ordinating Sectoral and Local Dimensions, in: Regional Studies, Vol. 29, No. 8, S. 779-784.

Fairley, J. (1996). Vocational Education and Training Reform in Scotland - Towards a Strategic Approach? In: Scottish Educational Review, Vol. 28, No. 1, S. 50-60.

Fairley, J./Lloyd, M. G. (1995). Scottish Enterprise and Highlands and Islands Enterprise: A Preliminary Assessment and Some Critical Questions for the Future, in: Regional Studies, Vol. 29, No. 6, S. 785-789.

Fuller, A./Unwin, L. (1998). Reconceptualising Apprenticeship: exploring the relationship between work and learning, in: Journal of Vocational Education and Training, Vol. 50, No. 2, S. 152-172.

Geißler, K. A. (1991). Das Duale System der industriellen Berufsausbildung hat keine Zukunft. In: Leviathan. Zeitschrift für Sozialwissenschaft, 19. Jg., S. 68-77.

General Teaching Council for Scotland (1995). What is the General Teaching Council for Scotland?, Edinburgh.

Gospel, H. F. (1998). Die Wiederbelebung des Lehrlingswesens: Fallstudien aus der Praxis in Großbritannien, in: Zeitschrift für Berufs- und Wirtschaftspädagogik, 94. Jg., H. 3, S. 340-363.

Greinert, W.-D. (1988). Marktmodell - Schulmodell - duales System. Grundtypen formalisierter Berufsbildung, in: Die berufsbildende Schule, 40. Jg., S. 145-156.

Greuling, O. (1996). Berufsvorbereitung und Berufswahl von britischen und deutschen Jugendlichen, Frankfurt a.M.

Gunning, D. (1999). A Modular, Outcome-based Qualifications System -The Scottish Experience (Paper presented at the GTZ-Tagung, 23.03.1999, Darmstadt), Edinburgh.

Harrison, C. (1997). How Scottish is the Scottish Curriculum: And Does it Matter? In: Clark, M. M.IMunn, P. (Eds.), Education in Scotland - Policy and Practice from Preschool to Secondary, London, S. 156-169.

Hilker, F. (1962). Vergleichende Pädagogik. Eine Einführung in ihre Geschichte, Theorie und Praxis, München.

Hodgson, A./Spours, K., Eds. (1997). Dearing and Beyond. 14-19 Qualifications, Frameworks and Systems, London.

Howieson, C. et al. (1997). Unifying Academic and Vocational Learning: The State of the Debate in England and Scotland, in: Journal of Education and Work, Vol. 10, No. 1, S. 5-35.

Humes, W./Bryce, T. (1999). The Distinctiveness of Scottish Education, in: Bryce, T./ Humes, W. (Eds.), Scottish Education, Edinburgh, S. 102-111.

Koch, R./Reuling, J. (1997). Institutionelle Rahmenbedingungen und Steuerung der beruflichen Erstausbildung am Beispiel von Deutschland, Frankreich und Großbritannien Beitrag zum Europäischen Berufsbildungsforschungsbericht, Thema I: Gesetzliche, institutionelle und politische Grundlagen der beruflichen Bildung (Endbericht), Berlin.

Kommission der Europäischen Gemeinschaften (1996). Weißbuch zur allgemeinen und beruflichen Bildung: Lehren und Lernen. Auf dem Weg zur kognitiven Gesellschaft, Luxemburg. 
Kommission der Europäischen Gemeinschaften/EUROSTAT/CEDEFOP (1997). Schlüsselzahlen zur Berufsbildung in der Europäischen Union, Luxemburg.

Mackinnon, D./Statham, J./Hales, M. (1996). Education in the UK: Facts and Figures, London.

Mädler, C./Pilz, M. (1999). Der „New Deal“ zur Bekämpfung der Jugendarbeitslosigkeit in Großbritannien, in: Erziehungswissenschaft und Beruf, 47. Jg., H. 2, S. 169-179.

Mansfield, B. (1990). Competence and Standards, in: Burke, J.W. (Ed.), Competency Based Education and Training, London, S. 26-38.

Marsden, D./Ryan, P. (1991). Initial Training, Labour Market Structure and Public Policy: Intermediate Skills in British and German Industry, in: Ryan, $P$. (Ed.), International Comparisons of Vocational Education and Training for Intermediate Skills, London, S. 251-285.

Michael, B. (1990). Veränderungen - Ein Brief aus Schottland, in: Grundschule, 22. Jg., H. 3, S 48-50.

Mitchell, J. (1995). Secondary education in Scotland [Council for Cultural Co-operation, Guide to secondary education in Europe], Strasbourg.

Münk, D. (1995). Kein Grund zur Eu(ro)phorie. Anmerkungen zu zentralen berufsbildungspolitischen Kontroversen des „Memorandums der Kommission über die Berufsausbildungspolitik der Gemeinschaft für die 90er Jahre“, in: Zeitschrift für Berufs- und Wirtschaftspädagogik, 91. Jg., S. 28-45.

Munn, P. (1997). Devolved Management of Schools, in: Clark, M. M./Munn, P. (Eds.), Education in Scotland - Policy and Practice from Pre-school to Secondary, London, S. 125-137.

Nitschke, W. (1986). Neue Entwicklungen im schottischen Schulwesen, in: Internationale Zeitschrift für Erziehungswissenschaft, 32. Jg., H. 1, S. 97-03.

Paterson, L. (1997). Policy-Making in Scottish Education: A Case of Pragmatic Nationalism, in: Clark, M. M./Munn, P. (Eds.), Education in Scotland - Policy and Practice from Pre-school to Secondary, London, S. 138-55.

Pilz, M. (1999 a). Modulare Strukturen in der beruflichen Bildung - eine Alternative für Deutschland? Eine explorative Studie am Beispiel des schottischen Modulsystems, Markt Schwaben.

Pilz, M. (1999 b). Schottland (Länderstudie), in: Lauterbach, U./Huck, W./Mitter, W. (Hrsg.), Internationales Handbuch der Berufsbildung, Baden-Baden.

Pilz, M. (1999 c). Die Modularisierung des schottischen Bildungssystems - Aktuelle Entwicklungen auf dem Weg zu einem einheitlichen System von allgemeiner und beruflicher Bildung, in: Die berufsbildende Schule, 51. Jg., H. 10, S. 329-334.

Pilz, M. (2000), Formen und Erkenntnisse der Modularisierung im schottischen Bildungssystem, in: Buschfeld, D. (Hrsg.), Moderate Modularisierung - eine nationale und internationale Differenzierungsstrategie? (Berufsbildung zwischen innovativer Programmatik und offener Umsetzung, Bd. 15), Bielefeld, S. 8-21 
Raffe, D. (1991). Scotland vs. England: The Place of ,Home Internationals “ in Comparative Research, in: Ryan, P. (Ed.), International Comparison of Vocational Education and Training for Intermediate Skills, London, S. 47-67.

Raffe, D. (1993). The Changing Scottish Scene: Implications for South of the Border, in: Richardson, W./Woolhouse, J./Finegold, D. (Eds.), The Reform of Post-16 Education and Training in England and Wales, Harlow, S. 54-73.

Raffe, D. (1997). Upper-Secondary Education, in: Clark, M. M./Munn, P. (Eds.), Education in Scotland - Policy and Practice from Pre-school to Secondary, London, S. 67-80.

Raffe, D. (1998). Does Learning Begin at Home? The Use of ,Home International ' Comparisons in UK Policy Making, in: Journal of Education Policy, Vol. 13, No. 5, S. 591-602.

Raffe, D. (2000). Investigating the Education Systems of the United Kingdom, in: Phillips, D. (Ed.), The Education Systems of the United Kingdom, Oxford, S. 9-28.

Raffe, D. et al. (1998 a). The Unification of Post-Compulsory Education: Towards a Conceptual Framework, in: British Journal of Educational Studies, Vol. 46, No. 2, S. 169-87.

Raffe, $D$. et al. (1998 b). Unifying Vocational and Academic Learning. Issues from a Comparison of England, Wales and Scotland, in: Mulder, M. (Ed.), European Vocational Education and Training Research (Proceedings of the VETNET Program at the ECER Conference on 17-20 September 1998 in Ljubljana, Slovenia), Enschede, S. 45-53.

Raffe, D. et al. (1999 a). Issues in a ,Home International ' Comparison of Policy Strategies: the experience of the Unified Learning Project, in: Coffield, F. (Ed.), ,Why's the beer always stronger up North?“ - Studies of lifelong learning in Europe, Bristol, S. 63-71.

Raffe, D. et al. (1999 b). Comparing England, Scotland, Wales and Nothern Ireland: The Case for 'Home Internationals' in Comparative Research, in: Comparative Education, Vol. 35, No. 1, S. 9-25.

Rat der Europäischen Gemeinschaften (1992). Richtlinie Nr. 92/51/EWG vom 18. Juni 1992 über eine zweite allgemeine Regelung zur Anerkennung beruflicher Befähigungsnachweise in Ergänzung zur Richtline 89/48/EWG, veröffentlicht am 24. Juli 1992, in: Amtsblatt der Europäischen Gemeinschaften, 35. Jg., Nr. L 209, S. 25-45.

Reuling, J. (1998). Modularisierung im englischen NVQ-System - ein Instrument zur Nachqualifizierung?, in: Davids, S. (Hrsg.), Modul für Modul zum Berufsabschluss-Berufsbegleitende Nachqualifizierung zwischen Flexibilität und Qualitätssicherung (Berichte zur beruflichen Bildung, H. 216), Bielefeld, S. 265-277.

Robertson-Wensauer, C. Y. (1994). Wie Bildungssystem und Selbstbewußtsein zusammengehören. Eine kulturhistorische Betrachtung am Beispiel Schottlands, in: Hettlage, $R$. (Hrsg.), Bildung in Europa: Bildung für Europa?, Regensburg, S. 93-104.

Ryan, P. (2001). Apprenticeship in Britain: tradition and innovation, in: Deißinger, Th. (Hrsg.), Berufliche Bildung zwischen nationaler Tradition und globaler Entwicklung Beiträge zur vergleichenden Berufsbildungsforschung, Baden-Baden [im Druck].

Saran, R. (1988). Schulpolitik unter der Regierung Thatcher, in: Die Deutsche Schule, 80. Jg., H. 4, S. 442-450. 
Schriewer, J. (1987). Vergleich als Methode und Externalisierung auf Welt: Vom Umgang mit Alterität in Reflexionsdisziplinen, in: Baecker, D. et al., Theorie als Passion, Frankfurt a.M., S. 629-668.

Scotland, J. (1969). The History of Scottish Education, London.

Scottish Enterprise (1997). Annual Report 1996/97, Glasgow.

Scottish Office (1995). Scottish Higher Education Institutions (Factsheet 4), Edinburgh.

Scottish Office (1996). Scottish Education (Factsheet 3), Edinburgh.

Scottish Parliament (2001). Official Reports, Vol. 1-10, Edinburgh

Scotvec (1995). Looking Ahead - Skillseekers, Glasgow.

Snell, K. D. M. (1996). The Apprenticeship System in British History: The Fragmentation of a Cultural Institution, in: History of Education, Vol. 25, S. 303-321.

Spours, K. et al. (1998). Unifying Post-Compulsory Education in England, Wales and Scotland: Conclusions of the Unified Learning Project (Unified Learning Project, Working Paper Eleven), Edinburgh.

Spours, K./Young, M. (1996). Dearing and Beyond: Steps and Stages to a Unified 14-19 Qualifications System?, in: British Journal of Education and Work, Vol. 9, No. 3, S. 5-8. SQA (1999). National Units: A Note on the Format, Glasgow.

Stipriaan, F. v./Lauterbach, U. (1995). Vereinigtes Königreich Großbritannien und Nordirland - England und Wales, in: Lauterbach, U./Huck, W.IMitter, W. (Hrsg.), Internationales Handbuch der Berufsbildung, Baden-Baden.

Twining, J. (1994). Das Berufsbildungssystem im Vereinigten Königreich, Berlin.

Unwin, L./Wellington, J. (1995). Reconstructing the Work-based Route: Lessons from the Modern Apprenticeship, in: The Vocational Aspect of Education, Vol. 47, S. 337-352.

Whitburn, J. (1995). Keeping the Register: Control over the Admission and Supply of Teachers in England and Wales, and Scotland, in: Compare, Vol. 25, No. 1, S. 49-57.

Williams, St./Raggatt, P. (1998). Contexualising Public Policy in Vocational Education and Training: The Origins of Competence-based Vocational Qualifications Policy in the UK, in: Journal of Education and Work, Vol. 11, No. 3, S. 275-292.

Wolf, A. (1995). Competence-Based Assessment, Buckingham.

\section{KURZBIOGRAPHIE:}

Prof. Dr. Thomas Deißinger, Diplom-Handelslehrer, geb. 1958; Professor für Wirtschaftspädagogik am Fachbereich Wirtschaftswissenschaften der Universität Konstanz; Studium der Wirtschaftspädagogik an der Universität Mannheim 1980-1985; Foreign Language Assistant in England 1985/1986; Wiss. Mitarbeiter/Assistent an der Universität Mannheim 1986-1997; Promotion 1991; Habilitation 1997; Vertretungsprofessur an der Universität Erlangen-Nürnberg 1997/1998.

Anschrift: Prof. Dr. Thomas Deißinger, Universität Konstanz, D127, 78457 Konstanz (E-Mail:Thomas.Deissinger@uni-konstanz.de) 
Dr. Matthias Pilz, Diplom-Handelslehrer, geb. 1968; Studienassessor an der Wirtschaftsschule Herrenhausen-Hannover und Lehrbeauftragter an der Universität Lüneburg; Ausbildung zum Bankkaufmann 1988-1990; Studium der Wirtschaftspädagogik an der Universität Göttingen 1990-1995; Wiss. Mitarbeiter an der TU Dresden 1996-1998; Promotion an der Universität Konstanz 1999; Referendariat 1998-2000.

Anschrift: Dr. Matthias Pilz, Hegebläch 31, 30419 Hannover (E-Mail: matthiaspilz@ hotmail.com) 